\title{
Electronic Health Records Communication among Team Members and Quality of Care and Costs for Patients with Cardiovascular Disease in Primary Care
}

\author{
Marlon P. Mundt \\ University of Wisconsin-Madison \\ marlon.mundt@fammed.wisc.edu
}

\author{
Larissa I. Zakletskaia \\ University of Wisconsin-Madison \\ larissa.zakletskaia@fammed.wisc.edu
}

\begin{abstract}
This study determines how changes in electronic health record (EHR) communication patterns in primary care teams are related to quality of care and costs for patients with cardiovascular disease. Counts of EHR messages routed between any two team members were extracted from the EHR system, and flow betweenness, the proportion of information passed indirectly within the team, was calculated. The analysis related changes in team flow betweenness to changes in acute care visits and associated medical costs for the teams' patients with cardiovascular disease. The results indicated that patient hospital visits increased by $7 \%$ (SE 3\%) for every 1\% increase in team EHR flow betweenness. Medical costs increased by $\$ 141$ (SE \$67) per patient for every 1\% increase in team EHR flow betweenness. EHR team communication flow patterns may be an important avenue to explore for raising quality of care and lowering costs for primary care patients with cardiovascular disease.
\end{abstract}

\section{Introduction}

Delivering the highest quality care for patients with cardiovascular disease (CVD), the leading cause of death, is a major public health issue. In the United States, about 610,000 people die from CVD annually, which is 1 in every 4 deaths [1]. CVD was associated with nearly $\$ 330$ billion in health care costs in 2014 [2]. About half of Americans (47\%) have at least one of the CVD risk factors (i.e., high blood pressure, high cholesterol or smoking) [3].

It is important to note that team-based hypertension management interventions in CVD care have demonstrated the largest effects in lowering blood pressure in contrast with other tested approaches to CVD care, such as patient education, clinician education, promotion of self-management, facilitated relay of clinical data, and financial incentives $[4,5]$. On the basis of strong evidence of effectiveness, the
Community Preventive Services Task Force recommends team-based care to improve blood pressure control, a risk factor for CVD [6].

Team-based primary care is crucial for optimal CVD care delivery in view of the fact that it would take a single physician 7.4 hours per day to deliver all recommended preventive care in light of over 2700 clinical recommendations, as well as 10.6 hours per day to manage chronic disease patient panels [7-9]. The solution to the impossibility of effectively delivering care to the average size US primary care panel of 2300 patients by a single physician lies with high-functioning primary care teams, which allow all team members to share in clinical care for patients with CVD [10-13].

To jointly care for patients with CVD, teams rely on their communication in the Electronic Health Record (EHR) which supports primary care teams as learning complex systems and allows them adaptability and flexibility in dealing with high-uncertainty clinical situations observed in the delivery of CVD care.

In high-functioning primary care teams, health professionals rely on team communication and actions coordinated among team members which provide the foundation of high quality care [14]. At the start of a primary care visit with a patient, a nurse takes vitals and asks for a visit reason. In this interaction, the nurse seeks information from the patient and assesses health problems. The nurse needs to decide what information (i.e., facts, insights, experience) to share with other team members in the EHR. Other team members will need to reflect and create understanding of the current patient health status, as well as establish mutual expectations about future patient health status. The manner in which this knowledge transfer in the EHR occurs will play a significant role in problem identification, assessment, sense making, and care management for the patient [15].

As has been documented in other complex systems outside of primary care (e.g., pilots and air traffic controllers, nuclear power plant operators) [16-19], data overload may overwhelm busy physicians with demands for time and cognitive resources. From this 
perspective, a physician, a nurse, a physician assistant, a medical assistant, a lab technician, and a receptionist could be as effective as their EHR dynamic interactions are. Communicating the right information to the right person at the right time is essential for high-performing teams. The relationship between EHR communication and quality of care is not trivial and it needs careful consideration.

Unfortunately, limited evidence exists on how patterns of team EHR communication contribute to quality of care and associated medical costs for patients with CVD. To put it differently, we lack clear understanding of how team EHR communication patterns relate to improvement or deterioration of CVD patient outcomes.

To fill this gap in the literature, our study investigates whether EHR communication patterns among primary care team members are associated with quality of care and associated medical cost for patients with CVD.

\section{Methods}

\subsection{Study setting and design}

The study data were drawn from 4 primary care clinics associated with a large health care system in southern Wisconsin. Practices invited to participate in the study were non-residency primary care clinics not involved in research or quality improvement initiatives at the time of inquiry. There were 2 urban, 1 suburban, and 1 rural primary care practices that agreed to participate in the study. The clinics have from 3 to 11 primary care providers (PCPs), defined as either a physician, nurse practitioner, or physician assistant, with average active patient panel sizes (at least 2 visits in the past 3 years) ranging from 987 to 1548 patients per PCP. The Institutional Review Board of the University of Wisconsin approved the study.

The study author initially introduced the study and provided study opt-out forms at an all-staff clinic meeting in the participating clinics. All physicians, physician assistants, nurse practitioners, registered nurses (RN), medical assistants (MA), licensed practical nurses (LPN), laboratory technicians, radiology technicians, clinic managers, medical receptionists, and other patient care staff were invited to participate. A trained researcher conducted a 30minute face-to-face structured survey about team communication in the clinic. The survey included a questionnaire on face-to-face communication with the other patient care staff at the clinic. Using a clinic staff roster as an aid for memory recall, participants were asked to identify, for each other employee at their clinic, how frequently they communicated face-to-face about patient care. These responses were used to calculate the presence or absence of communication network ties between all study team members. Study participants also completed the Warr-Cook-Wall job satisfaction survey [20]. Full details on the questionnaire are available in an initial study report [21]. Study participants consented to an analysis of their electronic communication patterns via the EHR. Communication patterns were analyzed based on counts of messages between any two team members, not on the content of the EHR messages. The sums of dyadic pair messages were used to create a square, sociometric matrix for each team.

\subsection{Clinical provider sample}

Eligibility criteria included 18 years of age or older, ability to read and understand English, and employment at the study clinic in a patient care or patient interaction capacity. Participants received \$10 for completing the study survey and were entered into a lottery drawing for $\$ 100$ with a 1 in 4 chance of winning. In addition, the researchers donated $\$ 200$ to a local community outreach program of the clinic's choice to study clinics with $90 \%$ subject participation. Over 97\% (83 of 85 invited) of eligible participants took part in the study.

\subsection{CVD patient panel sample}

An EHR search linked primary care teams to their patients with cardiovascular disease age 21 and older seen by the team over the 18-month study period from July 1, 2013 to December 31, 2014. To ensure continuity of care, the active patient panel consisted of patients who had at least one visit with the PCP in the past 18 months, and at least 2 visits in the past 36 months. Patients with visits to multiple PCPs were assigned to the PCP whom they saw most frequently, or, in the case of a tie, to the PCP seen at the most recent visit. Cardiovascular disease diagnoses were determined by the presence of 2 validated ICD- 9 codes for hypertension, congestive heart failure, coronary artery disease, heart attack, arrhythmia, cerebrovascular disease, or stroke (4010-4019, 4280042802, 41401, 4300-4389, 4109, 42789) on 2 separate occasions within the past 3 years.

\subsection{Team membership survey}

To determine team membership, health professionals were asked to consider a team definition and indicate on a full clinic staff roster who is on their 
care team. The care team was defined as 'the smallest unit of individuals within the clinic that care for a specific patient panel.' Care team membership included a PCP (physician, nurse practitioner, or physician assistant) and all clinic employees who indicated on the survey that they belonged to that PCP's care team. Finally, any individual whom the PCP named as a care team member was also included in the care team.

Clinic staff members could be included on multiple care teams based on responses to the team membership question. For example, a nurse could indicate belonging to the care team of multiple PCPs. In this case, care team memberships would overlap as the nurse would be considered a member of each PCPled team.

The study sought to examine changes in team EHR communication structure that were not a direct result of staff turnover; teams with staff departures during the study were excluded from the analysis. The initial sample included 24 care teams. A total of 5 teams were excluded due to significant turnover during the study period. In two of the care teams, the physicians left the clinic during the 18-month followup. In the other 3 excluded care teams, team members who were involved in more than $25 \%$ of the team communications left the clinic. The sample size for the study after excluding teams with staff turnover during the 18 -month study period was 19 primary care teams at 4 primary care clinics.

\subsection{Team EHR communication}

Electronic communication about patient care between team members is sent and received through the secure Epic (Epic Systems Corp., Madison, Wisconsin) EHR system employed at each study clinic. Each clinic employee is assigned an EHR message account for transmitting patient care information to other team members. A log file stores the sender and receiver for each EHR message sent. In this study, we extracted counts of EHR messages sent between each member to every other team members through the secure electronic messaging function (Inbox) of the EHR. A communication connection was deemed present between two team members if either of them sent an EHR message to the other person. These established communication connections created an EHR communication network which was presented as a sociomatrix for the study analysis [22]. Due to HIPAA constraints, other forms of electronic communication, such as email, Skype for Business, alerts/notes, and forwarded messaging to/from patients were not available for the analysis and were not analyzed, and as such are left to future research.
EHR communication between all members of the primary care team was extracted from the EHR log file by totaling the number of messages between each dyadic pair (a directed to-from pair of care team members) for each time period of the study. Message counts between team members were totaled for three 6month time periods: (1) July 1, 2013-December 31, 2013; (2) January 1, 2014-June 30, 2014; and (3) July 1, 2014-December 31, 2014. The sum of dyadic pair messages produced a sociometric matrix for each team and each time period. The sociometric team matrix data were then used to compute social network analysis measures of EHR communication within the team for each time period.

The analysis calculated the following measures: (1) the total number of messages sent within the team for each 6-month period; (2) the percentage of the team's messages sent to the study team PCP; and (3) the social network analysis measure of flow betweenness for the EHR team communication network [23, 24]. Flow betweenness is the amount of information which travels between team members indirectly, by going through another team member within the team, as opposed to being sent directly from person to person. Flow betweenness quantifies the number of times one node acts as a bridge along the shortest path between two other nodes. It has been introduced as a measure for quantifying the control of one individual on the communication between other members in a social network.

To calculate flow betweenness for the whole EHR communication network, the analysis begins by computing the flow betweenness for each vertex in the network. Vertex flow betweenness is the amount of message information transferred through the vertex $\mathrm{i}$ when the maximum flow of information is averaged over all pairs a and $b$ in the network [25, 26]. Normalizing the individual flow betweenness in the network by the total number of messages transmitted yields the team-based measure of flow betweenness which can be expressed as a percentage.

\subsection{EHR health utilization data for patients with cardiovascular disease}

Number of emergency department (ED) visits and of hospital visit days for team patients with CVD were extracted from the EHR as utilization counts over the 18-month study period. Healthcare costs were calculated by applying average healthcare costs derived from published reports to health care utilization counts. An average cost of US\$664 per emergency department visit and US\$1628 per hospital day was applied to each recorded visit [27, 28]. 
Patient age, gender, race/ethnicity, insurance status, tobacco use, and available EHR diagnoses of chronic conditions were drawn from the EHR to control for patient panel characteristic differences across study teams.

\subsection{Statistical analysis}

This study analysis examines the association between changes in team flow betweenness and changes in patient health care utilization and cost outcomes over time for the teams' patients with CVD. Flow betweenness changes were calculated as the difference in team flow betweenness between time period 3 and time period 2, and the difference in team flow betweenness between time period 2 and time period 1. Changes in flow betweenness were then modeled as predictors of changes in patient utilization and costs for the teams' patients with CVD using multilevel mixed effects modeling (MLME) [29]. The MLME analysis used a log link function for count outcomes (ED visits, hospital days) and a normal link function for medical costs. MLME nests patients by care teams and care teams within clinics to estimate the association between changes in flow betweenness and changes in cardiovascular disease outcomes while adjusting for team-level confounders, such as team size, average number of years in the clinic, average job satisfaction, and team face-to-face communication density, as well as clinic-level clustering. Density is the ratio of communication ties observed to the total number of possible network connections. In a dense network, information can flow quickly between team members. By using difference scores in the model, the analysis controls for omitted variables which are constant over time, such as the physical proximity of team members to each other in the clinic setting and the experience and training of the team. The analyses used Ucinet 6 [30] for constructing networks and obtaining social network measurements, and used HLM 7.0 [31] for constructing hierarchical models.

To control for differences in patient panel characteristics across teams, the study adjusted for patient age, gender, race/ethnicity, insurance status, tobacco use, and available EHR diagnoses of chronic conditions referenced in the U.S. Centers for Medicare and Medicaid Services Chronic Condition Warehouse (e.g., asthma, cancer, chronic kidney disease, chronic obstructive pulmonary disease, depression, osteoarthritis, osteoporosis, rheumatoid arthritis) or in the Charlson Comorbidity Index (e.g., dementia, peptic ulcer disease). The Charlson Comorbidity Index was also included to adjust for potential confounding by multiple simultaneous chronic conditions [32].

\section{Results}

This study included 19 primary care teams from 4 primary care clinics. A total of 83 health professionals participated in the study (Table 1). Study participants included 19 PCPs (14 physicians, 5 nurse practitioners or physician assistants), 19 RNs, 21 MAs or LPNs, 10 medical receptionists, 10 laboratory or radiology technicians, and 4 clinic managers.

Table 1. Study sample

\begin{tabular}{|c|c|c|}
\hline \multicolumn{3}{|c|}{$\begin{array}{c}\text { Clinician Sample Characteristics } \\
\text { ( } n=83 \text { employees at } 4 \text { primary care clinics) }\end{array}$} \\
\hline \multicolumn{2}{|c|}{ Job Position in Clinic } & $\%(n)$ \\
\hline \multicolumn{2}{|c|}{ Physician (MD/DO) } & $16.9 \%(14)$ \\
\hline \multicolumn{2}{|c|}{$\begin{array}{r}\text { Nurse Practitioner or Physician } \\
\text { Assistant }\end{array}$} & $6.0 \%(5)$ \\
\hline \multicolumn{2}{|c|}{ Clinic Manager } & $4.8 \%(4)$ \\
\hline \multicolumn{2}{|c|}{ Registered Nurse } & $22.9 \%(19)$ \\
\hline \multicolumn{2}{|c|}{$\begin{array}{r}\text { Licensed Practical Nurse or Medical } \\
\text { Assistant }\end{array}$} & $25.3 \%(21)$ \\
\hline \multicolumn{2}{|c|}{ Laboratory or Radiology Technician } & $12.0 \%(10)$ \\
\hline \multicolumn{3}{|c|}{ Gender } \\
\hline & Female & $95.2 \%(79)$ \\
\hline \multicolumn{2}{|l|}{ Years at Clinic } & \\
\hline \multicolumn{2}{|c|}{1 year of less } & $14.4 \%(12)$ \\
\hline \multicolumn{2}{|c|}{$>1$ to 3 years } & $33.7 \%(28)$ \\
\hline \multicolumn{2}{|c|}{$>3$ to 6 years } & $20.4 \%(17)$ \\
\hline \multicolumn{2}{|c|}{$>6$ to 10 years } & $9.6 \%(8)$ \\
\hline \multicolumn{2}{|c|}{$>10$ years } & $21.7 \%(18)$ \\
\hline \multicolumn{3}{|l|}{ \% Full Time Employment } \\
\hline \multicolumn{2}{|c|}{$50 \%$ or less } & $10.8 \%(9)$ \\
\hline \multicolumn{2}{|c|}{$>50 \%$ to $75 \%$} & $14.4 \%(12)$ \\
\hline \multicolumn{3}{|c|}{\begin{tabular}{|l|l|}
$>75 \%$ & $74.7(62)$ \\
\end{tabular}} \\
\hline \multicolumn{3}{|c|}{$\begin{array}{c}\text { Patient Sample Characteristics } \\
\text { (n=5,154 patients with CVD seen at } 4 \text { primary care } \\
\text { clinics) }\end{array}$} \\
\hline \multirow[t]{2}{*}{ Age, mean (sd) } & \multicolumn{2}{|c|}{$59.8(13.0)$} \\
\hline & \multicolumn{2}{|l|}{$\%(n)$} \\
\hline Female & \multicolumn{2}{|c|}{$48.0(2474)$} \\
\hline \multicolumn{3}{|l|}{ Race/Ethnicity } \\
\hline Non-Hispanic White & \multicolumn{2}{|c|}{$89.7(4623)$} \\
\hline Black & \multicolumn{2}{|c|}{$2.9(149)$} \\
\hline White Hispanic & \multicolumn{2}{|c|}{$3.6(186)$} \\
\hline Asian & \multicolumn{2}{|l|}{$1.6(82)$} \\
\hline Other/Missing & \multicolumn{2}{|c|}{$2.2(114)$} \\
\hline \multicolumn{3}{|l|}{ Insurance } \\
\hline Commercial & $49.8(25)$ & \\
\hline Medicare & $44.4(22 \varepsilon$ & \\
\hline Medicaid & $3.9(201)$ & \\
\hline
\end{tabular}




\begin{tabular}{|l|l|}
\hline \multicolumn{1}{|c|}{ Uninsured } & $1.9(98)$ \\
\hline Tobacco use & $14.4(742)$ \\
\hline $\begin{array}{l}\text { Charlson Co-Morbidity } \\
\text { Index, mean (sd) }\end{array}$ & $2.8(1.8)$ \\
\hline $\begin{array}{l}\text { Health Care Use (per } \\
\text { patient per past 6 months) }\end{array}$ & mean (sd) \\
\hline Emergency Department visits & $0.15(1.16)$ \\
\hline Hospital days & $0.54(4.33)$ \\
\hline $\begin{array}{l}\text { Medical costs (US\$ per } \\
\text { patient per past 6 months) }\end{array}$ & $\$ 983(\$ 7,632)$ \\
\hline
\end{tabular}

Study participants were $95 \%$ female, which is in line with U.S. Census Bureau data indicating that $91 \%$ of all nurses, nurse practitioners, and licensed practice nurses, and $97 \%$ of all medical receptionists, are female [33]. Fourteen percent of the participants had worked at their practice for one year or less and one quarter worked $75 \%$ of full-time equivalent or less.

Care teams ranged in size from 11 to 28 individuals, averaging 18 team members (Table 2). While team PCPs spearheaded a single care team, other team members (RNs, LPNs/MAs, etc.) belonged to multiple care teams. On average, clinic staff other than the PCP belonged to 4 care teams.

The teams' panels of patients with CVD consisted of 5,154 patients. Over half of the patients were women (55\%), most were non-Hispanic white (92\%), and most had access to private insurance (53\%) or Medicare (39\%). Comorbidities were common among the patients, with $10 \%$ diagnosed with asthma and $23 \%$ diagnosed with diabetes. EHR records showed that patients had on average 0.2 emergency department visits and 0.5 hospital visit days per 6 months. Acute medical care costs averaged US\$983 per patient per 6 months.

As seen in Table 2, the number of EHR messages sent between team members averaged 4,099 messages in a 6-month period. Over 80 percent of EHR messages were sent from a team member to the PCP. Team flow betweenness averaged $4.6 \%$, indicating that just under 5 percent of information in the EHR network was passed indirectly (e.g., forwarded message) from one team member to another before reaching its final destination.

Figure 1 visually represents the EHR interaction networks in two study teams. The team in Panel A of Figure 1 was in the lowest quartile of health care utilization and costs and had a less dense communication network than the team in Panel B of Figure 1, which was in the highest quartile of health care utilization and costs. Team B had visibly more EHR communication connections among more team members. Team A demonstrated flow betweenness of
$2.3 \%$, which was in the lowest quartile of team flow betweenness. Team B, on the other hand, had flow betweenness of $10.6 \%$, which was in the highest quartile of team flow betweenness. As evidenced in the figures, Team B which had more information passed indirectly by more team members (e.g., message forwarding) in the EHR network and had higher health care utilization and associated medical costs for their cardiovascular disease patient panels.

Table 2. EHR communication networks in primary care teams ( $n=19$ care teams)

\begin{tabular}{|l|l|}
\hline \multicolumn{1}{|c|}{ Characteristics } & \multicolumn{1}{c|}{ Mean (sd) } \\
\hline Team size & $17.6(5.4)$ \\
\hline $\begin{array}{l}\text { EHR messages sent within team per 6 } \\
\text { months }\end{array}$ & $4099(2462)$ \\
\hline EHR messages to team PCP (\%) & $81.4(13.6)$ \\
\hline Team flow betweenness (\%) & $4.6(2.8)$ \\
\hline $\begin{array}{l}\text { Face-to-face communication density } \\
\text { (\%) }\end{array}$ & $47.3(13.4)$ \\
\hline
\end{tabular}

${ }^{\mathrm{a}}$ Flow betweenness=amount of information which travels between team members indirectly; ${ }^{b}$ Density=percentage of the communication ties present in the team divided by all possible ties which could be present

Table 3 presents results from MLME evaluation of change in EHR team communication pattern (i.e., change in flow betweenness) in relation to change in frequencies of EHR-documented patient ED visits and hospital days and associated health care costs, while adjusting for team size, team-level face-to-face communication density (percentage of the communication ties present in the team divided by all possible ties which could be present), average number of years in the clinic among team members, average job satisfaction among team members, and patientlevel confounding (age, gender, race/ethnicity, medical insurance, tobacco use, chronic disease, Charlson Comorbidity Index). The results show that increases in team EHR flow betweenness (e.g., message forwarding) were associated with increased patient emergency department visits, more hospital days and higher associated medical costs. For every 1 percentage point increase in team EHR flow betweenness, there was a corresponding 2.7\% (SE $1.2 \%)$ increase in emergency department visits per patient per 6 months, 7.4\% (SE 3.4\%) more hospital days, and \$141 (SE \$67) higher medical costs per patient per 6 months. Baseline team face-to-face communication density was not associated with changes in health care utilization or cost. 
A. Visual representation of EHR messaging network within a primary care team with lowest quartile of flow betweenness (2.3\%) and lowest quartile of patient healthcare utilization and costs

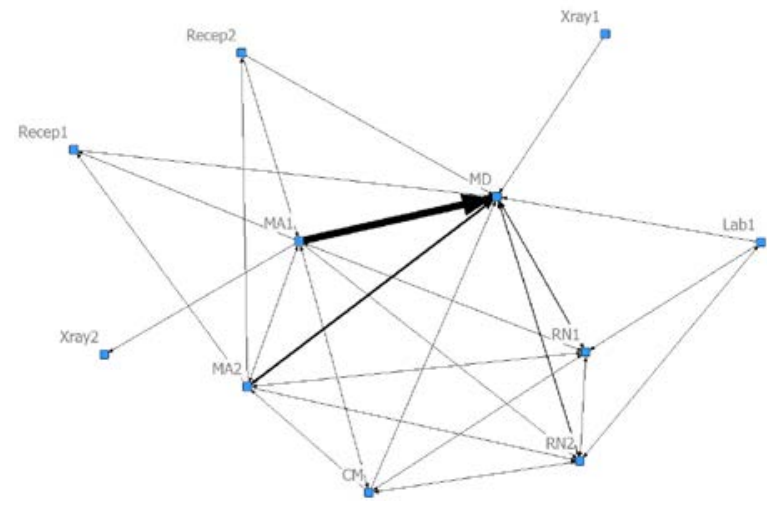

B. Visual representation of EHR communication within a primary care team with highest quartile of flow betweenness (10.6\%) and upper quartile of patient healthcare utilization and costs

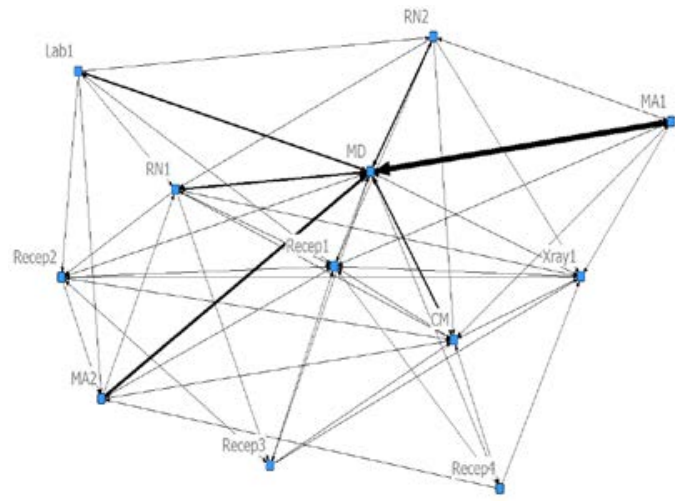

Figure 1. Primary care team electronic health record (EHR) communication networks

Table 3. MLME model of changes in EHR communication and patient outcomes, adjusted for patient characteristics ( $N=19$ teams, $n=2,242$ patients)

\begin{tabular}{|c|c|c|c|c|c|c|}
\hline & \multicolumn{2}{|c|}{$\begin{array}{l}\text { Emergency Department } \\
\text { Visits }\end{array}$} & \multicolumn{2}{|c|}{ Hospital Days } & \multicolumn{2}{|c|}{ Healthcare Costs (\$) } \\
\hline & $\beta(\mathrm{SE})$ & $p$-value & $\beta$ (SE) & p-value & $\beta(\mathrm{SE})$ & p-value \\
\hline \multicolumn{7}{|l|}{ Clinical Team Sample } \\
\hline $\begin{array}{l}\text { EHR team messaging } \\
\text { flow betweenness }\end{array}$ & $0.027(0.012)$ & $0.024^{*}$ & $0.074(0.034)$ & $0.027^{*}$ & $141(67)$ & $0.036^{*}$ \\
\hline $\begin{array}{l}\text { Face-to-face } \\
\text { communication density }\end{array}$ & $-0.079(0.397)$ & 0.843 & $0.412(1.277)$ & 0.747 & $62(226)$ & 0.783 \\
\hline Team size & $0.010(0.007)$ & 0.188 & $0.033(0.024)$ & 0.167 & $60(42)$ & 0.154 \\
\hline Team job satisfaction & $0.008(0.014)$ & 0.580 & $0.013(0.044)$ & 0.769 & $27(79)$ & 0.736 \\
\hline Team years in clinic & $-0.017(0.027)$ & 0.532 & $0.017(0.087)$ & 0.848 & $16(154)$ & 0.919 \\
\hline \multicolumn{7}{|l|}{ CVD Patient Panel } \\
\hline Male & $0.033(0.030)$ & 0.277 & $0.040(0.097)$ & 0.680 & $86(171)$ & 0.614 \\
\hline \multicolumn{7}{|l|}{ Age } \\
\hline $50-64$ & $0.155(0.074)$ & $0.021^{*}$ & $0.461(0.208)$ & $0.016^{\star}$ & $856(286)$ & $0.013^{*}$ \\
\hline$\geq 65$ & $0.171(0.048)$ & $0.001^{*}$ & $0.575(0.176)$ & $0.003^{\star}$ & $1056(412)$ & $0.002^{*}$ \\
\hline \multicolumn{7}{|l|}{ Race/Ethnicity } \\
\hline White & $0.022(0.093)$ & 0.816 & $-0.067(0.299)$ & 0.822 & $97(531)$ & 0.855 \\
\hline Black/African American & $-0.003(0.127)$ & 0.978 & $-0.419(0.410)$ & 0.307 & $-676(727)$ & 0.353 \\
\hline Asian & $-0.069(0.160)$ & 0.666 & $0.293(0.516)$ & 0.570 & $-532(916)$ & 0.561 \\
\hline Hispanic & $0.053(0.139)$ & 0.706 & $-0.100(0.449)$ & 0.824 & $-123(796)$ & 0.877 \\
\hline Medicare & $0.075(0.067)$ & 0.263 & $0.448(0.217)$ & $0.039 *$ & $782(384)$ & $0.042^{*}$ \\
\hline Medicaid & $0.024(0.103)$ & 0.820 & $0.026(0.333)$ & 0.937 & 81 (591) & 0.891 \\
\hline Private Insurance & $-0.015(0.069)$ & 0.828 & $0.183(0.221)$ & 0.408 & 291 (392) & 0.458 \\
\hline Tobacco use & $-0.026(0.042)$ & 0.535 & $-0.137(0.134)$ & 0.306 & $242(238)$ & 0.309 \\
\hline $\begin{array}{l}\text { Charlson Comorbidity } \\
\text { Index }\end{array}$ & $0.027(0.012)$ & $0.025^{\star}$ & $0.070(0.039)$ & 0.071 & $132(68)$ & 0.054 \\
\hline
\end{tabular}




\section{Discussion}

This study evaluates the connection between EHR communication among primary care team members and ED visits and hospital stay days and associated medical costs for the patients with CVD. Our findings demonstrate that variations in EHR team communication are associated with statistically significant differences in ED visits and hospital stay days for the patients with CVD.

Our results show that patients with CVD experienced better care if their care team engaged in fewer indirect EHR connections to share patient care information. This finding demonstrates that CVDrelated care outcomes may depend on the timeliness of passing patient care information to the appropriate team member via shortest path in the EHR communication network.

In contrast, teams that forwarded EHR messages more often than other teams and shared patient care information indirectly among team members had more ED visits and longer hospital stay days for their patients with CVD. It is conceivable that these teams may have insufficient and incomplete understanding of unfolding situations if the team members rely on indirect EHR communication to reach the right recipient. Indirect EHR communication may also suggest that the team members may not rely efficiently on face-to-face communication to problem solve the issues at hand in the context of clinical uncertainty. A study key informant highlighted the importance of fewer EHR connections to guard against cognitive overload: "People get so overwhelmed with all the emails that they, they blow by them, don't read them and that communication, important information, is lost."

In addition, indirect EHR communication patterns among team members may demonstrate a lack of team cognition which is cognitive information processing on the part of the team (e.g., team-level problem-solving, sense-making). The link between team cognition and team functioning is well-established in the literature [34, 35]. Team cognition (i.e., team shared understanding of the capabilities of each team member, who is good at what, who should be assigned what at what time) allows a team to be more than the sum of its parts, affords teams flexibility and adaptability and enables teammates to relate to each other and to newly emergent information in the process of task performance. A study key informant emphasized that overreliance on EHR messaging is detrimental for team cognition: "I think we communicate so much through the electronic medical record that we forget to be human and interact that way.” Future studies may wish to explore if EHR communication patterns could be indicative of team functioning as a team.

Furthermore, our results indicate that primary care teams are complex learning healthcare systems driven by dynamic interactions among the team members who engage with each other and relate to their environment in nonlinear fashion, who self-organize their collective efforts in the process of CVD care delivery, resulting in unpredictable, emergent creative sense-making team behavior rather than adhere to a linear set of prescribed processes [36, 37]. From this vantage point, to raise CVD-related care delivered by complex learning healthcare systems such as primary care teams, we need to promote changes in EHR communication among all team members. Future studies may wish to explore further how EHR communication patterns could be optimized to gain highest quality of CVD care at lowest medical costs.

Notably, a consideration should be given to a range of sociotechnical issues associated with the effectiveness of EHR use for CVD-related care among team members, such as EHR interface design and fit of EHR capabilities with clinical workflow and clinician CVD-related communication needs. A sociotechnical systems approach to designing CVD care assisted EHR will support team cognition in the clinical context of workload, team member roles and shared responsibilities, and workflow [38]. Achieving such an EHR design requires studying team members and their interactions in the clinical context and environment of shared roles and responsibilities in CVD team care model with a purpose of developing a deep understanding of the team shared decision making involved in CVD care delivery. Future studies may choose to take a sociotechnical systems approach [3941] to EHR design capable of supporting CVD-related care communication among team members.

To summarize, our study results suggest that better understanding of EHR communication among team members may allow raising quality of care for patients with CVD at low cost. EHR communication in teams may also serve as an indicator of team cognitive ability to process information and act upon it at the team level (i.e., team cognition) which has been linked to team performance outcomes [42]. Improving EHR communication patterns among team members may be a low-hanging fruit which could help raise CVD patient outcomes without significant amounts of additional investment, as EHR adoption and implementation is underway in primary care clinics nationwide.

\subsection{Limitations}


The study should be viewed in light of its limitations. First, this study does not support a causal pathway between team EHR communication among team members and health care utilization and associated medical costs for patients with CVD due to endogeneity concerns of the data. Experimental study designs are needed to uncover the causal mechanisms between team EHR communication patterns and CVDrelated patient care. Second, the study data come from 4 practices in the Midwest, so the results may not be generalizable to a broader national context. Third, the study looked only at frequency of EHR communication among team members and did not evaluated communication content, clinical care context or timeliness of information sharing among team members. Future research is needed to directly address the link between team EHR communication patterns and quality of CVD care delivery. Finally, the study did not investigate why different team members choose a particular mode of communication (i.e., face-to-face vs EHR) to coordinate patient care.

\section{Conclusions}

This study highlights that EHR communication among team members is associated with CVD care outcomes and associated medical costs in primary care. Patients with CVD may experience better quality of care if their primary care teams engage in a focused EHR communication among team members characterized by timely, frequent EHR communication between the appropriate teammates, which discourages message forwarding of patient care related information among team members.

\section{References}

[1] Kochanek KD, Xu J, Murphy SL, Minino AM, Kung HC. Deaths: Final Data for 2009. Hyattsville, MD: National Center for Health Statistics;2012.

[2] Benjamin EJ, Virani SS, Callaway CW, et al. Heart Disease and Stroke Statistics-2018 Update: A Report From the American Heart Association. Circulation. Mar 20 2018;137(12):e67-e492.

[3] Fryar CD, Chen T-C, Li X. Prevalence of uncontrolled risk factors for cardiovascular disease: United States, 19992010. NCHS Data Brief. 2012(103):1-8.

[4] Walsh JM, McDonald KM, Shojania KG, et al. Quality improvement strategies for hypertension management: a systematic review. Medical care. Jul 2006;44(7):646-657.

[5] Carter BL, Rogers M, Daly J, Zheng S, James PA. The potency of team-based care interventions for hypertension: a meta-analysis. Arch Intern Med. Oct 26 2009;169(19):17481755.

[6] Community Preventive Services Task Force. Team-based care to improve blood pressure control: recommendation of the Community Preventive Services Task Force. American journal of preventive medicine. Jul 2014;47(1):100-102.

[7] Institute of Medicine (U.S.). Committee on Standards for Developing Trustworthy Clinical Practice Guidelines., Graham R. Clinical practice guidelines we can trust. Washington, DC: National Academies Press; 2011.

[8] Yarnall KS, Pollak KI, Ostbye T, Krause KM, Michener JL. Primary care: is there enough time for prevention? American journal of public health. Apr 2003;93(4):635-641.

[9] Ostbye T, Yarnall KS, Krause KM, Pollak KI, Gradison $\mathrm{M}$, Michener JL. Is there time for management of patients with chronic diseases in primary care? Annals of family medicine. May-Jun 2005;3(3):209-214.

[10] Andreatta PB. A typology for health care teams. Health care management review. Oct-Dec 2010;35(4):345-354.

[11] Chesluk BJ, Holmboe ES. How teams work--or don't--in primary care: a field study on internal medicine practices. Health affairs. May 2010;29(5):874-879.

[12] Schuetz B, Mann E, Everett W. Educating health professionals collaboratively for team-based primary care. Health affairs. Aug 2010;29(8):1476-1480.

[13] Grumbach K, Bodenheimer T. Can health care teams improve primary care practice? Jama. Mar 10 2004;291(10):1246-1251.

[14] Cain C, Haque S. Organizational Workflow and Its Impact on Work Quality. In: Patient Safety and Quality: An Evidence-Based Handbook for Nurses. Edited by Hughes RG. Rockville (MD); 2008.

[15] Moody J, White DR. Structural cohesion and embeddedness: A hierarchical concept of social groups. Am Sociol Rev 2003, 68(1):103-127.

[16] Sarter NB, Schroeder B. Supporting decision making and action selection under time pressure and uncertainty: The case of in-flight icing. Human factors 2001, 43(4):573-583.

[17] Smith PJ, McCoy E, Layton C. Brittleness in the design of cooperative problem-solving systems: The effects on user performance. Ieee T Syst Man Cy A 1997, 27(3):360-371.

[18] Endsley MR, Bolté B, Jones DG. Designing for situation awareness: an approach to user-centered design. London; New York: Taylor \& Francis; 2003.

[19] Hoffman RR, Militello LG. Perspectives on cognitive task analysis: historical origins and modern communities of practice. New York: Psychology Press; 2009. 
[20] Warr P, Cook J, Wall T. Scales for the Measurement of Some Work Attitudes and Aspects of Psychological WellBeing. J Occup Psychol. 1979;52(2):129-148.

[21] Mundt MP, Gilchrist VJ, Fleming MF, Zakletskaia LI, Tuan WJ, Beasley JW. Effects of Primary Care Team Social Networks on Quality of Care and Costs for Patients with Cardiovascular Disease. Annals of family medicine. Mar-Apr 2015;13(2):139-148.

[22] Wasserman S, Faust K. Social network analysis: methods and applications. Cambridge; New York: Cambridge University Press; 1994.

[23] Freeman LC, Borgatti SP, White DR. Centrality in Valued Graphs - a Measure of Betweenness Based on Network Flow. Soc Networks. Jun 1991;13(2):141-154.

[24] Newman MEJ. A measure of betweenness centrality based on random walks. Soc Networks. Jan 2005;27(1):3954.

[25] Scott J, Carrington PJ. The SAGE handbook of social network analysis. London; Thousand Oaks, Calif.: SAGE; 2011.

[26] Cormen TH, Leiserson CE, Rivest RL. Introduction to Algorithms. Cambridge: MIT Press; 2014.

[27] Agency for Healthcare Research and Quality. Expenses for a Hospital Emergency Room Visit, 2003. 2006; http://meps.ahrq.gov/mepsweb/data_files/publications/st111/ stat111.pdf. Accessed May 6, 2014.

[28] Beckers Hospital Review. Average cost per inpatient day across 50 states in 2010. 2010; http://www.beckershospitalreview.com/lists/average-costper-inpatient-day-across-50-states-in-2010.html. Accessed May 6, 2014.

[29] Raudenbush SW, Bryk AS. Hierarchical linear models: applications and data analysis methods. 2. ed. Thousand Oaks: Sage Publ.; 2010.

[30] Borgatti SP, Everett MG, Freeman LC. UCINET 6 for Windows Software for social network analysis. Lexington, KY: Analytic Technologies: University of Greenwich; 2002.

[31] Raudenbush SW, Bryk AS, Cheong YF, Congdon RT, DuToit M. HLM7 hierarchical linear and nonlinear modeling. Skokie, IL: SSI; 2011.
[32] Charlson ME, Pompei P, Ales KL, Mackenzie CR. A New Method of Classifying Prognostic Co-Morbidity in Longitudinal-Studies - Development and Validation. Journal of chronic diseases. 1987;40(5):373-383.

[33] National Center for Health Workforce Analysis (U.S.). The U.S. Health Workforce Chartbook. Rockville, Md.: U.S. Dept. of Health and Human Services, Health Resources and Services Administration Bureau of Health Professions, National Center for Health Workforce Analysis; 2013.

[34] DeChurch LA, Mesmer-Magnus JR. Measuring shared team mental models: A meta-analysis. Group Dynamics: Theory, Research, and Practice 2010, 14(1):1-14.

[35] Cooke NJ, Gorman JC, Myers CW, Duran JL. Interactive team cognition. Cogn Sci 2013, 37(2):255-285.

[36] Young RA, Roberts RG, Holden RJ. The Challenges of Measuring, Improving, and Reporting Quality in Primary Care. Annals of family medicine 2017, 15(2):175-182.

[37] McDaniel RR, Jr., Lanham HJ, Anderson RA. Implications of complex adaptive systems theory for the design of research on health care organizations. Health care management review 2009, 34(2):191-199.

[38] Institute of Medicine (U.S.). Committee on Patient Safety and Health Information Technology. Health IT and patient safety: building safer systems for better care. Washington, D.C.: National Academies Press; 2012.

[39] Sittig DF, Singh H. A new sociotechnical model for studying health information technology in complex adaptive healthcare systems. Qual Saf Health Care 2010, 19 Suppl 3:i68-74.

[40] Berg M. Patient care information systems and health care work: a sociotechnical approach. Int J Med Inform 1999, 55(2):87-101.

[41] Harrison MI, Koppel R, Bar-Lev S. Unintended consequences of information technologies in health care--an interactive sociotechnical analysis. J Am Med Inform Assoc 2007, 14(5):542-549.

[42] DeChurch LA, Mesmer-Magnus JR. The cognitive underpinnings of effective teamwork: a meta-analysis. The Journal of applied psychology 2010, 95(1):32-53. 